\title{
Perfil, produção e avaliação de participantes das Américas e África em uma especialização sobre drogas*
}

\author{
Margarita Antonia Villar Luis ${ }^{1}$ \\ (iD) https://orcid.org/0000-0002-9907-5146 \\ Alisséia Guimarães Lemes ${ }^{2}$ \\ (D) https://orcid.org/0000-0001-6155-6473 \\ Deivson Wendell da Costa Lima ${ }^{3}$ \\ (iD) https://orcid.org/0000-0002-7020-2172 \\ Liliane Santos da Silva ${ }^{1}$ \\ (iD) https://orcid.org/0000-0001-8639-874X \\ Vagner Ferreira do Nascimento 4 \\ (iD) https://orcid.org/0000-0002-3355-163X
}

\footnotetext{
A publicação deste artigo na Série Temática "Recursos Humanos em Saúde e Enfermagem" se insere na atividade 2.2 do Termo de Referência 2 do Plano de Trabalho do Centro Colaborador da OPAS/OMS para o Desenvolvimento da Pesquisa em Enfermagem, Brasil.

1 Universidade de São Paulo, Escola de Enfermagem de Ribeirão Preto, Centro Colaborador da OPAS/OMS para o Desenvolvimento da Pesquisa em Enfermagem, Ribeirão Preto, SP, Brasil.

2 Universidade Federal de Mato Grosso, Campus Universitário do Araguaia, Barra do Garças, MT, Brasil.

${ }^{3}$ Universidade do Estado do Rio Grande do Norte, Faculdade de Enfermagem, Mossoró, RN, Brasil.

4 Universidade do Estado de Mato Grosso, Campus Tangará da Serra, Tangará da Serra, MT, Brasil.
}

Objetivo: identificar perfis, produção e avaliação dos profissionais das Américas e da África em Cursos de Especialização em drogas. Método: estudo retrospectivo documental, baseado em dados secundários, através das fichas de registro de todas as versões dos Cursos de Especialização para formação de pesquisadores em álcool e outras drogas psicoativas (2002 a 2015), promovido pela Escola de Enfermagem de Ribeirão Preto da Universidade de São Paulo. Resultados: houve a participação de 258 pessoas, sendo $62 \%$ da América do Sul e 38\% da América do Norte, América Central e África Portuguesa. A maioria do sexo feminino (77,0\%), faixa etária de 30 a 59 anos $(81,8 \%)$, com mestrado $(51,2 \%)$, da área de ciências da saúde $(79,5 \%)$ e atuando como docentes universitários (82,6\%). Dos participantes, 176 finalizaram o curso com publicações e submissões de trabalhos científicos. $\mathrm{Na}$ avaliação final, a maioria referiu satisfação com o curso e que indicaria para outros profissionais. Considerações finais: o estudo apresentou os resultados de uma experiência exitosa e organizada, oferecida pela enfermagem brasileira, na capacitação de pesquisadores, proporcionando a ampliação e disseminação do conhecimento científico sobre o fenômeno das drogas por mais de uma década, tanto em território nacional como em outros países da América e África.

Descritores: Capacitação Profissional; Educação de Pósgraduação; Pesquisadores; Enfermagem Psiquiátrica; Saúde Mental.

\section{Como citar este artigo}

Luis MAV, Lemes AG, Lima DWC, Silva LS, Nascimento VF. Profile, production and evaluation of participants from the Americas and Africa in a specialization on drugs. SMAD, Rev Eletrônica Saúde Mental Álcool Drog. 2021 jul.-set.;17(3):67-77. doi: https://dx.doi.org/10.11606/issn.1806-6976.smad.2021.175834 


\section{Profile, production and evaluation of participants from the Americas and Africa in a specialization on drugs}

Objective: to identify the profile, production and evaluation of professionals from the Americas and Africa in Specialization Courses on drugs. Method: a retrospective and documentary study, based on secondary data, using the registration forms of all versions of the Specialization Courses to train researchers in alcohol and other psychoactive drugs (2002 to 2015), promoted by the Ribeirão Preto Nursing School, University of Sao Paulo. Results: a total of 258 individuals participated, $62 \%$ from South America and 38\% from North America, Central America and Portuguese Africa. The majority were female $(77.0 \%)$, aged 30 to 59 years old $(81.8 \%)$, with a master's degree $(51.2 \%)$, from the health sciences area (79.5\%), and working as university professors (82.6\%). Of the participants, 176 completed the course with publications and submissions of scientific papers. In the final assessment, most reported satisfaction with the course and that they would recommend it to other professionals. Final considerations: the study presented the results of a successful experience, organized and offered by Brazilian Nursing, in the training of researchers, providing the expansion and dissemination of scientific knowledge about the drug phenomenon for more than a decade, both in the national territory and in other American and African countries.

Descriptors: Professional Training; Education, Graduate; Research Personnel; Psychiatric Nursing; Mental Health.

\section{Perfil, producción y evaluación de participantes de Américas y África en una especialización en drogas}

Objetivo: identificar perfil, producción y evaluación de profesionales de las Américas y África en Cursos de Especialización en Drogas. Método: estudio retrospectivo y documental, basado en datos secundarios, con la utilización de los formularios de inscripción de todas las versiones de los Cursos de Especialización para la formación de investigadores en alcohol y otras drogas psicoactivas (2002 a 2015), promovido por la Escuela de Enfermería de Ribeirão Preto Universidad de San Pablo. Resultados: participaron 258 personas, 62\% de Sudamérica y 38\% de Norteamérica, Centroamérica y África portuguesa. La mayoría eran mujeres (77,0\%), de 30 a 59 años $(81,8 \%)$, con grado de maestría $(51,2 \%)$, del área de ciencias de la salud $(79,5 \%)$ y profesoras universitarias $(82,6 \%)$. De los participantes, 176 completaron el curso con publicaciones y envío de artículos científicos. En la evaluación final, la mayoría reportó satisfacción con el curso y afirmó que lo recomendaría a otros profesionales. Consideraciones finales: el estudio presentó los resultados de una experiencia exitosa y organizada, ofrecida por la enfermería brasileña, en la formación de investigadores y proporcionó la expansión y difusión del conocimiento científico sobre el fenómeno de las drogas durante más de una década, tanto en el territorio nacional como en otros países de América y África.

Descriptores: Capacitación Profesional; Educación de Postgrado; Investigadores; Enfermería Psiquiátrica; Salud Mental. 


\section{Introdução}

A United Nations Office on Drugs and Crime (UNODC), em seu relatório mundial sobre drogas, aponta que em 2018 cerca de 269 milhões de pessoas no mundo referiram ter usado drogas psicoativas pelo menos uma vez no ano anterior. $E$, estima-se que mais de 35 milhões sofrem com algum transtorno desencadeado pelo consumo, com limitações ao acesso e disponibilidade a serviços de tratamento ${ }^{(1)}$.

Esse consumo, cada vez maior, amplia alguns problemas sociais, como violência, acidentes e comportamentos de risco, bem como pode levar à dependência química(2-3). Isso gera importantes repercussões nas esferas administrativas, jurídicas e de políticas públicas, como saúde, educação e justiça(4). Assim, torna-se necessário promover ações que considerem a pessoa e suas relações com a droga, com atenção aos aspectos singulares e contextuais.

Sabe-se que a intervenção em âmbito escolar e acadêmico já revela caminho promissor em prol da redução do consumo e/ou maior conscientização sobre as drogas e seus efeitos ${ }^{(5-7)}$, porém há necessidade de capacitação dos profissionais que vivenciam o fenômeno das drogas em seu cotidiano, em especial daqueles que atuam na área da saúde, seja no processo de formação/ graduação ou na condição de profissional inserido nos serviços que atendem tais demandas ${ }^{(8)}$, como preconizado pela Lei no 11.343/2006, que rege a política pública sobre drogas $^{(9)}$.

Para isso, estratégias vêm sendo estabelecidas em articulação com universidades e órgãos governamentais, visando o fortalecimento de ações que promovam investimentos em pesquisas e treinamentos na área de álcool e/ou outras drogas ${ }^{(10-12)}$. Ainda assim, há muitas transformações e inovações técnico-científicas para serem implementadas nesse contexto(13), já que as abordagens práticas em relação à vulnerabilidade das pessoas para o uso e abuso de drogas não têm avançado significativamente ${ }^{(4)}$; circunstância que impulsiona a criação de propostas de capacitações na área.

Diante disso, a Escola de Enfermagem de Ribeirão Preto da Universidade de São Paulo (EERP-USP) em convênio com a Organização dos Estados Americanos (OEA), a Comissão Interamericana para o Controle dos Estados Americanos (CICAD) e a Secretaria Nacional de Políticas Sobre Drogas (SENAD) ofereceu o curso de pósgraduação lato sensu para formação de pesquisadores em álcool e outras drogas psicoativas. Este curso teve como propósitos disseminar conhecimentos sobre o fenômeno das drogas; estimular e ampliar a produção científica em âmbito nacional e internacional, bem como possibilitar trocas de experiências entre os participantes da área da saúde e afins, de países e continentes distintos.
O potencial e alcance de cursos dessa natureza podem influir nas políticas públicas e fundamentar novas práticas de cuidados ${ }^{(14)}$, principalmente porque em vários contextos de formação (graduação) de enfermagem, a carga horária de disciplinas voltadas à saúde mental e psiquiatria e que contemplem o uso de drogas e dependência química está se reduzindo ao longo dos $\operatorname{anos}^{(15)}$.

Diante disso, é fundamental conhecer melhor o perfil dos profissionais que trabalham na rede de formação e pesquisa em álcool e outras drogas, e que direta ou indiretamente também participam do processo de atenção à saúde dessa população(16). Outrossim, o próprio Ministério da Saúde (MS) vem estimulando avaliações contínuas de egressos, processos de trabalho e de ensinoaprendizado de cursos de graduação e pós-graduação (lato e stricto sensu) $)^{(17)}$. Isso exposto, o objetivo deste estudo foi identificar perfil, produção e avaliação dos profissionais das Américas e da África em Cursos de Especialização em drogas.

\section{Método}

Trata-se de estudo retrospectivo, documental e quantitativo, baseado em dados secundários. Foi realizado no período de agosto de 2019 a junho de 2020, a partir das fichas de registro das nove versões do curso de Formação de Pesquisadores em Álcool e Outras Drogas Psicoativas (2002 a 2015), preenchidas pelos participantes e pela coordenação do curso no momento da inscrição (aspectos sociodemográfico, perfil de formação e profissão); instrumentos de avaliação final do curso (conteúdo do curso, material, pessoal de apoio e avaliação geral do curso); e relatórios enviados às instituições que deram apoio financeiro e logístico aos participantes (produção científica).

A coleta de dados foi realizada no acervo do laboratório de pesquisa "Stress, álcool e drogas" da EERPUSP, mediante a autorização da coordenadora responsável pelas edições do curso. Foram incluídas no estudo todas as fichas disponíveis no acervo, sendo 258 fichas de inscrições, 219 fichas de avaliação e nove relatórios finais de curso.

Após a finalização da coleta de dados, procedeu-se à digitação dupla dos dados em planilha eletrônica do programa Microsoft Excel 2013, que posteriormente foram confrontados no Data Compare. Na sequência, o banco de dados foi importado para o STATA versão 13.1, com análise estatística descritiva, apresentando em números absolutos e relativos, por meio de gráficos e tabelas. Os dados relacionados ao eixo temático da produção científica gerada a partir do curso foram agrupados por área central dos trabalhos.

O estudo respeitou todos os aspectos éticos em pesquisa, de acordo com a Resolução 466/12 e 510/2016 
do Conselho Nacional de Saúde (CNS), tendo aprovação do Comitê de Ética em Pesquisa com Seres Humanos da EERP-USP.

\section{Resultados}

\section{A organização do curso}

O curso Formação de Pesquisadores em Álcool e Outras Drogas Psicoativas oferecido pela EERP-USP, através do departamento de Enfermagem Psiquiátrica, modalidade lato sensu, teve nove edições, entre os anos de 2002 a 2015.

Cada edição do curso ocorreu em um período de 12 meses, com carga horária total de $600 \mathrm{~h}$, estruturado em quatro módulos, sendo os três primeiros à distância (450 h) e o último presencial (150 h), que foram realizados nas dependências da EERP/USP. No módulo I, abordou as especificidades do uso da plataforma e busca de literatura científica; no módulo II, a temática abordada foram as drogas psicoativas nas sociedades contemporâneas; no módulo III, discutiu-se sobre a metodologia de investigação frente ao fenômeno das drogas psicoativas; módulo IV, presencialmente, abordou-se os projetos de pesquisas aplicados ao fenômeno das drogas.

O curso era destinado a docentes universitários e profissionais atuantes nos Ministérios de Educação,
Saúde e Justiça e que, adicionalmente, tivessem algum conhecimento ou experiência em pesquisa ou que atuassem em áreas relacionadas ao uso de drogas lícitas e ilícitas, residentes em países da América e África Portuguesa.

Para a seleção dos participantes, a EERP-USP e a SENAD divulgaram as etapas de inscrição do curso entre as instituições de forma eletrônica em páginas oficiais e via contato por telefone e e-mail de gestores.

Os conteúdos teóricos e exercícios incluídos no curso foram elaborados nos idiomas português e espanhol. Entre as nove edições já ofertadas deste curso, houve a participação de 258 pessoas, distribuídas entre os anos de 2002 a 2015, com maior participação nos anos de 2005 (33) e 2015 (40).

\section{Caracterização dos participantes}

Do total de participantes, $62 \%$ pertenceram a países do continente da América do Sul e outros 38\% somam os países pertencentes à América do Norte, América Central e África Portuguesa. Quanto à distribuição por países, em sua maioria foram pessoas oriundas do Brasil (48), México (46), Colômbia (20), Chile (17), Peru (17) e Nicarágua (15). Já os países do continente africano apresentam pouca participação nas edições do curso (11) (Figura 1).

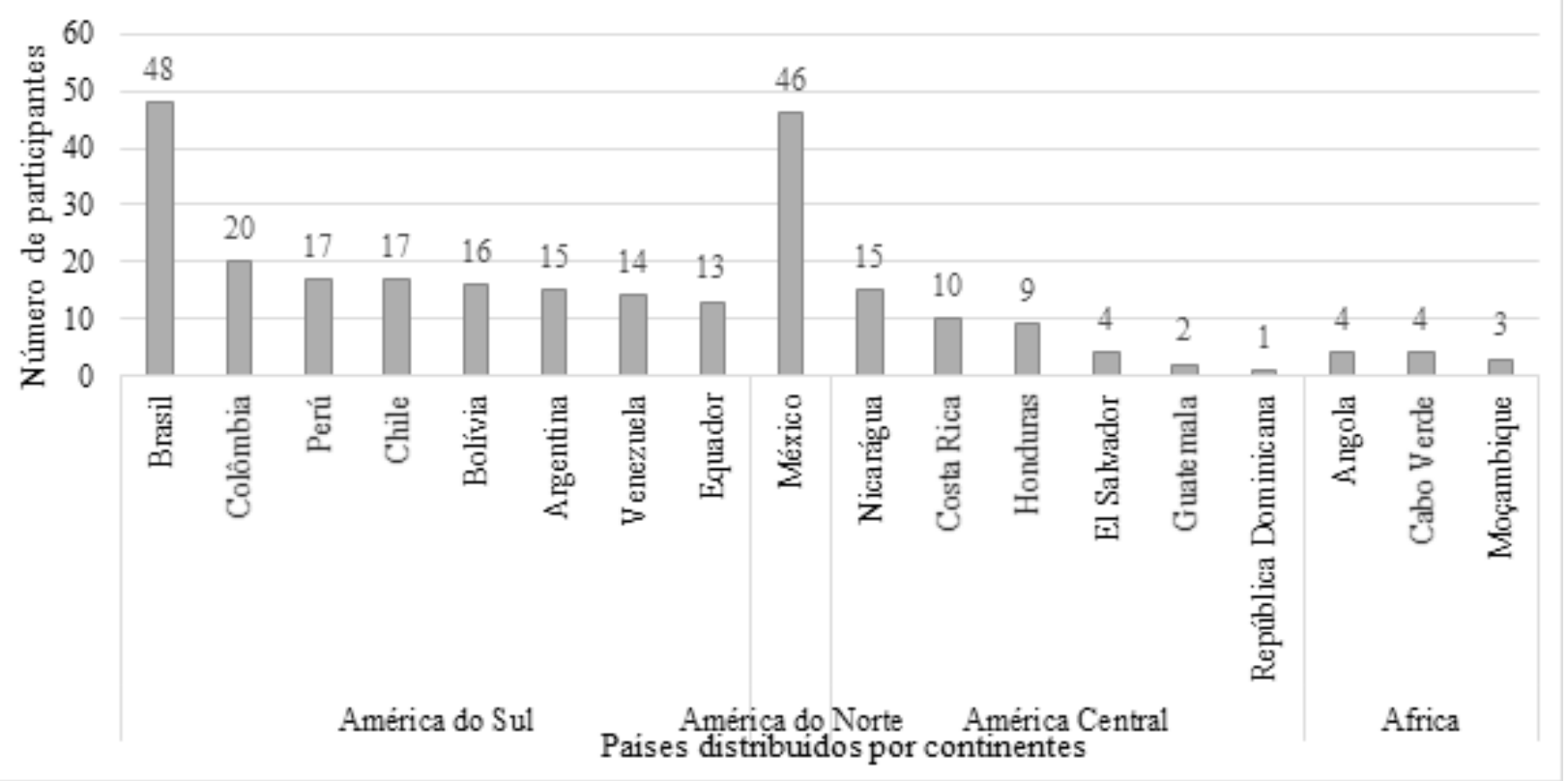

Fonte: Acervo documental da EERP-USP, 2020

Figura 1 - Distribuição de participantes do curso de Formação de Pesquisadores em Álcool e outras Drogas, por continente e país. Ribeirão Preto, SP, Brasil, 2002-2015 (n=258)

A Tabela 1 destaca os aspectos sociodemográficos dos participantes do curso. A maioria foi do sexo feminino
$(77,0 \%)$, com idade entre 30 a 59 anos $(81,8 \%)$, e titulação de mestre $(51,2 \%)$. 
Tabela 1 - Distribuição dos aspectos sociodemográficos de participantes do curso de Formação de Pesquisadores em Álcool e outras Drogas. Ribeirão Preto, SP, Brasil, 2002-2015 $(n=258)$

\begin{tabular}{llcc}
\hline Características & & $\boldsymbol{n}$ & $\%$ \\
\hline \multirow{2}{*}{ Sexo } & Feminino & 198 & 77,0 \\
& Masculino & 60 & 23,0 \\
\hline \multirow{2}{*}{ Faixa etária } & $24-30$ & 39 & 15,1 \\
& $30-40$ anos & 75 & 29,1 \\
& $40-50$ & 65 & 25,2 \\
& 50 -60 anos & 71 & 27,5 \\
& $\geq 60$ anos & 08 & 3,1 \\
\hline \multirow{2}{*}{ Grau de escolaridade } & Graduação & 66 & 25,6 \\
& Especialização & 31 & 12,0 \\
& Mestrado & 132 & 51,2 \\
& Doutorado & 29 & 11,2 \\
\hline
\end{tabular}

Fonte: Acervo documental da EERP-USP, 2020

Em relação à formação e profissão dos participantes, seguindo a classificação de área de formação proposta pela Coordenação de Aperfeiçoamento de Pessoal de Nível Superior (CAPES), destaca-se na Tabela 2 que 79,5\% dos profissionais eram da área de ciências da saúde, com formação em enfermagem $(67,8 \%)$ e medicina $(9,3 \%)$. Enquanto 8,9\% pertenciam à área de ciências humanas, com formação em psicologia $(8,1 \%)$ e pedagogia $(6,2 \%)$.

Quanto ao vínculo institucional, $84,5 \%$ eram trabalhadores de universidade e $11,2 \%$ de órgãos governamentais. Em sua maioria, atuavam como docentes universitários $(82,5 \%)$ (Tabela 2$)$.

Tabela 2 - Distribuição dos aspectos de formação e profissão dos participantes do curso de Formação de Pesquisadores em Álcool e outras Drogas. Ribeirão Preto, SP, Brasil, 2002-2015 $(n=258)$

\begin{tabular}{|c|c|c|c|}
\hline Descrição & & $n$ & $\%$ \\
\hline \multirow{6}{*}{$\begin{array}{l}\text { Área de } \\
\text { conhecimento } \\
\text { de formação } \\
\text { (Graduação) }\end{array}$} & Ciências da saúde & 205 & $79,5 \%$ \\
\hline & Ciências humanas & 23 & $8,9 \%$ \\
\hline & Ciências sociais & 11 & $4,3 \%$ \\
\hline & Ciências exatas & 02 & $0,8 \%$ \\
\hline & Ciências biológicas & 01 & $0,4 \%$ \\
\hline & Outras ciências & 16 & $6,2 \%$ \\
\hline \multirow{7}{*}{$\begin{array}{l}\text { Área de } \\
\text { formação } \\
\text { (Graduação) }\end{array}$} & Enfermagem & 175 & 67,8 \\
\hline & Medicina & 24 & 9,3 \\
\hline & Psicologia & 21 & 8,1 \\
\hline & Pedagogia & 16 & 6,2 \\
\hline & Serviço Social & 06 & 2,3 \\
\hline & Direito & 03 & 1,2 \\
\hline & Outras formações & 13 & 5,1 \\
\hline \multirow{4}{*}{$\begin{array}{l}\text { Vínculo } \\
\text { institucional }\end{array}$} & Universidade & 218 & 84,5 \\
\hline & Órgão governamental & 29 & 11,2 \\
\hline & Instituição de saúde & 08 & 3,1 \\
\hline & Outros & 03 & 1,2 \\
\hline
\end{tabular}

(continua...)

\begin{tabular}{llcc}
\hline Descrição & & $\boldsymbol{n}$ & $\%$ \\
\hline & Docente universitário & 213 & 82,6 \\
& Enfermeiro & 9 & 3,5 \\
& Psicólogo & 8 & 3,1 \\
Ocupação & Assessor técnico da SENAD* & 5 & 1,9 \\
& Médico & 4 & 1,6 \\
& Assessor técnico da saúde & 3 & 1,2 \\
& Polícia de investigação criminal & 2 & 0,8 \\
& Assistente social & 2 & 0,8 \\
& Técnico em saúde mental & 2 & 0,8 \\
& Terapeuta ocupacional & 1 & 0,4 \\
& Estatístico & 1 & 0,4 \\
& Farmacêutico & 1 & 0,4 \\
& Outras ocupações & 7 & 2,5
\end{tabular}

Fonte: Acervo documental da EERP-USP, 2020

${ }^{*}$ SENAD $=$ Secretaria Nacional de Políticas Sobre Drogas

\section{Produção científica dos participantes}

Uma das propostas do curso foi incentivar os participantes a divulgarem suas pesquisas científicas geradas a partir das disciplinas e experiências profissionais. Assim, é possível verificar que uma parte significativa dos participantes $(n=176)$ publicou ao menos um trabalho $(n=166)$ ou submeteu manuscritos à apreciação de revistas $(n=10)$ brasileiras e internacionais. Quanto à produção científica, os eixos temáticos surgiram do agrupamento conforme o foco central dos trabalhos, predominando o eixo "Uso de drogas" (36,3\%), "Prevenção à saúde" (21,5\%) e "Crenças relacionadas ao uso de drogas" (19,0\%) (Tabela 3).

Tabela 3 - Distribuição dos eixos temáticos da produção científica dos participantes do curso de Formação de Pesquisadores em Álcool e outras Drogas. Ribeirão Preto, SP, Brasil, 2002-2015 ( $n=176)$

\begin{tabular}{llcc}
\hline Descrição & & N & $\%$ \\
\hline \multirow{2}{*}{ Produção científica } & Artigos publicados & 166 & 94,3 \\
& Artigos submetidos & 10 & 5,7 \\
\hline & Uso de drogas & 64 & 36,3 \\
& Prevenção a saúde & 38 & 21,5 \\
$\begin{array}{l}\text { Eixos temáticos } \\
\text { da produção } \\
\text { científica }\end{array}$ & Crenças & 33 & 19,0 \\
& Tratamento & 23 & 13,0 \\
& Educação & 11 & 6,2 \\
\hline
\end{tabular}

Fonte: Acervo documental da EERP-USP, 2020

As revistas selecionadas pelos pesquisadores foram da área de enfermagem $(92,6 \%)$ ou em outras revistas $(7,4 \%)$; com foco de publicação em periódicos A1 $(60,8 \%), B 2(10,2 \%), B 1(3,0 \%), B 4(2,4 \%), A 2$ 
(1,8\%) e B3 e B5 (2,0\% ambos) e outros 33 artigos foram publicados em livro (19,8\%). A maioria dos participantes publicou ou submeteu seus manuscritos em revistas brasileiras $(95,7 \%)$, espanhola $(3,1 \%)$, costarriquenha e colombiana $(0,6 \%$ cada); publicados em espanhol $(62,0 \%)$, português $(25,3 \%)$ e inglês $(12,7 \%)$.

\section{Avaliação dos participantes}

Em todas as edições dos cursos, os participantes eram convidados a preencher voluntariamente a avaliação do curso após sua conclusão. Dos 258 participantes de todas as edições realizadas (2002-2015), 219 avaliaram o curso, uma adesão de $85 \%$ dos participantes.

Quanto à avaliação dos participantes, $88 \%$ se mostraram satisfeitos com o desenvolvimento do curso, seja em relação a sua duração ( $88 \%)$, aos equipamentos oferecidos ou utilizados ( $88 \%$ ) e/ou ao atendimento do pessoal de apoio (95\%). Um total de $94 \%$ dos participantes recomendaria o curso para outros profissionais (Tabela 4).

Tabela 4 - Distribuição dos participantes do curso de Formação de Pesquisadores em Álcool e outras Drogas, quanto à avaliação do curso. Ribeirão Preto, SP, Brasil, 2002-2015 $(n=219)$

\begin{tabular}{|c|c|c|c|c|c|}
\hline \multirow{2}{*}{ Descrição } & & \multicolumn{2}{|c|}{ Sim } & \multicolumn{2}{|c|}{ Não } \\
\hline & & $n$ & $\%$ & $n$ & $\%$ \\
\hline \multirow{3}{*}{$\begin{array}{l}\text { Conteúdo } \\
\text { do curso }\end{array}$} & $\begin{array}{l}\text { Duração do curso } \\
\text { adequada }\end{array}$ & 193 & 88 & 26 & 12 \\
\hline & $\begin{array}{l}\text { Continuidade e organização } \\
\text { adequadas }\end{array}$ & 179 & 82 & 40 & 18 \\
\hline & $\begin{array}{l}\text { Nível e objetivos propostos } \\
\text { adequados }\end{array}$ & 179 & 82 & 40 & 18 \\
\hline \multirow{3}{*}{ Material } & Equipamentos adequados & 193 & 88 & 26 & 12 \\
\hline & $\begin{array}{l}\text { Clareza, instrução e boa } \\
\text { qualidade dos materiais } \\
\text { didáticos }\end{array}$ & 190 & 87 & 29 & 13 \\
\hline & Quantidade suficientes & 188 & 86 & 31 & 16 \\
\hline \multirow{2}{*}{$\begin{array}{l}\text { Pessoal } \\
\text { de apoio }\end{array}$} & $\begin{array}{l}\text { Atendimento da secretaria } \\
\text { adequado }\end{array}$ & 210 & 96 & 09 & 04 \\
\hline & $\begin{array}{l}\text { Eficiência e rapidez no } \\
\text { atendimento }\end{array}$ & 208 & 95 & 11 & 05 \\
\hline \multirow{3}{*}{$\begin{array}{l}\text { Avaliação } \\
\text { geral do } \\
\text { curso }\end{array}$} & Recomendação do curso & 206 & 94 & 13 & 06 \\
\hline & Expectativa alcançada & 199 & 91 & 20 & 09 \\
\hline & Adequação da infraestrutura & 193 & 88 & 26 & 12 \\
\hline
\end{tabular}

Fonte: Acervo documental da EERP-USP, 2020

De forma complementar, ao responderem sobre recursos recebidos, verificou-se que poucos tiveram apoio financeiro substancial para o desenvolvimento de investigações, principalmente participantes do Brasil e do México. Outros tiveram pequenas ajudas locais ou de órgãos internacionais como a Organização PanAmericana da Saúde (Argentina, Nicarágua, Honduras). Os participantes do Chile e da Colômbia relataram não possuir apoio financeiro e os provenientes dos demais países não deram resposta a esse respeito.

\section{Discussão}

O curso de formação de pesquisadores em álcool e outras drogas, em nível de especialização, contou com subsídio financeiro das instituições envolvidas para o custeio local (Brasil), com disponibilidade de infraestrutura e suporte ao desenvolvimento e apoio aos participantes (hospedagem, alimentação e material de consumo) durante a fase presencial.

Houve maior participação de brasileiros, que provavelmente vislumbraram como viável a etapa/módulo presencial no Brasil e por reconhecerem a experiência e alcance da EERP-USP na área de enfermagem psiquiátrica. A pouca participação de membros de outros países possivelmente ocorreu devido à dificuldade desses profissionais em serem liberados de seus trabalhos, para o cumprimento do momento presencial do curso realizado no Brasil e pela disponibilidade reduzida dessas instituições no fomento à capacitação dos seus colaboradores.

Nas edições do curso, houve predomínio de participantes do sexo feminino, o que pode estar relacionado com uma presença maciça de mulheres matriculadas em cursos da saúde, em especial na enfermagem ${ }^{(18-20)}$. Por outro lado, também se relaciona ao crescimento de mulheres na ciência, na conquista de importantes espaços em instituições e na produção científica(21-22).

A temática abordada no curso (uso de drogas), além de constituir uma área de interesse de vários pesquisadores e ser abordada em muitas formações na área de saúde mental e enfermagem ${ }^{(16,23)}$, está presente no cotidiano da sociedade, inclusive dentro de muitos contextos profissionais e acadêmicos ${ }^{(24)}$.

Estudiosos desta temática apontam ainda que a inserção de conteúdo relacionado ao uso de drogas nos currículos de enfermagem é fundamental, pois pode ser uma estratégia para melhorar as habilidades desses profissionais e a qualidade do atendimento aos usuários de drogas que procuram assistência, nas mais diferentes especialidades médicas ${ }^{(16,25)}$. Visto que, a alta prevalência e as consequências relacionadas com o consumo de álcool e outras drogas podem ser minimizadas com um trabalho coletivo e intersetorial na base da formação e nos anos subsequentes, aperfeiçoamentos e pós-graduação(23,26).

Outro dado a ser destacado é a participação de mestres e doutores nas edições deste curso. Isso vem ao encontro com a necessidade de qualificação destes profissionais, que buscam ampliar e aperfeiçoar seu campo de atuação e pesquisa, seja no âmbito universitário, como em todos os níveis de assistência nos serviços de saúde ${ }^{(27)}$. Além disso, cada vez mais, tem-se exigido dos profissionais de enfermagem múltiplas competências que atendam às necessidades da comunidade, com ou sem dependências químicas, mas que requer cuidado atento, humanizado e baseado em evidências científicas ${ }^{(23,28)}$. 
Adicionalmente, qualificações na área de saúde mental e de drogas psicoativas permitem a qualificação da equipe, já que esses profissionais assumem a função de coordenadores do cuidado no enfrentamento das drogas, na aptidão do cuidado especializado e qualidade do ensino em saúde ${ }^{(26)}$ e promovem reflexões constantes sobre as práticas, por meio da educação permanente ${ }^{(29-30)}$, o que responde e supre também, as necessidades do mercado de trabalho nesse setor, atraindo e dando oportunidades de empregabilidade aos profissionais ${ }^{(31)}$.

No estudo, a presença de enfermeiros em todas as edições do curso indica a efetiva participação da enfermagem no campo da saúde mental, quer seja na área da docência ou assistencial(32). Essa grande adesão de enfermeiros pode estar relacionada ao fato de que esses profissionais se encontram melhor posicionados na identificação, acompanhamento e intervenção das pessoas que estão em risco ao uso de álcool e outras drogas (27). Outro aspecto pode ser a necessidade desses profissionais se manterem continuamente em aperfeiçoamento frente às novas abordagens e estratégias de prevenção, promoção, reabilitação e ressocialização(26), além de que participar de cursos desta natureza traz impacto positivo no comportamento do profissional, através da modificação de suas crenças pessoais sobre drogas(25).

Diante do cenário atual, onde o número de usuários em situação de uso problemático de drogas é crescente, há um apelo mundial para o desenvolvimento e fortalecimento de especialistas que colaborem para o desenvolvimento de novas tecnologias e estratégias de cuidado(33-36). Para tanto, a formação desses profissionais, na área de álcool e/ou outras drogas psicoativas, deve também avançar na identificação dos tipos de substâncias existentes, efeitos e formas de prevenção(28). Deve-se aperfeiçoar o acolhimento e reconhecimento das especificidades clínicas bem como suas histórias de vida, relações sociais e singularidades do usuário e família, propondo como prioridade estabelecer a autonomia, liberdade e consciência dos riscos/danos, sobretudo sobre seu corpo e sua saúde, para então ser possível compreender a relação entre pessoa, sofrimento e o uso dessas substâncias ${ }^{(28)}$.

Nesse sentido, a oportunidade de congregar profissionais de vários países em formação sobre álcool e/ou outras drogas no Brasil gerou a possibilidade de complementariedade de saberes, pela interação positiva entre diversas formas de apreender, assistir e cuidar dos usuários. Ao mesmo tempo, realçou o potencial e envergadura de cursos dessa modalidade no panorama científico e educativo.

No âmbito científico, o curso teve como proposta a descoberta de evidências, desenvolvimento de habilidades e produtos, o que estimulou a produção e publicação de várias investigações, baseadas nas experiências dos participantes, e pôde contribuir com a melhoria da prática no cuidado do usuário de drogas e na dinâmica de trabalho dos profissionais de saúde. Ainda que o incentivo do exercício profissional baseado em evidências não seja uma prioridade em todos os serviços de saúde, as organizações que incentivam e investem nesse desenvolvimento humano, político e científico se aproximam de práticas mais seguras e de qualidade(37).

Em relação aos trabalhos científicos gerados no curso, percebeu-se o interesse principalmente no eixo "uso de drogas". Esse tema de estudo possibilita conhecer o perfil dos usuários e seu consumo, criar tecnologias, repensar métodos de prevenção focados nas singularidades(38) e na reabilitação psicossocial, em diferentes níveis de cuidado(39). No entanto, estudo recente com egressos de uma especialização em saúde da família, predominantemente cubanos, apontou que suas produções não alcançaram 1\% sobre a temática saúde mental e áreas correlacionadas (40\%). Isso reforça a premissa de que o direcionamento de um curso possui estreita relação com o perfil dos participantes e produção gerada.

Outro estudo revelou que pesquisadores da área da enfermagem integram o grupo de precursores de pesquisas e produção científica na área de prevenção de drogas $^{(41)}$ e são os mais presentes em especializações na área da saúde no Brasil(16). Isso aproxima da realidade encontrada no presente estudo, em que esses profissionais lideraram na participação e no volume de produção.

Quanto à avaliação dos profissionais com o curso, de modo geral, mostraram-se satisfeitos com o conteúdo, material, pessoal de apoio, e na avaliação geral do curso. Em 2014, um curso de prevenção do uso de drogas promovido pela SENAD com duração de oito meses, também teve avaliação bastante positiva pelos participantes, indicando que o método de ensino e estratégias utilizadas se mostraram adequados ao público capacitado $^{(42)}$. Em outras capacitações em Minas Gerais (BR) sobre álcool e outras drogas para profissionais da saúde e assistência social, apesar da avaliação positiva da proposta pedagógica, houve questionamentos sobre alguns momentos de ênfase teórica que, segundo os participantes, apareceram descolados das realidades práticas dos serviços ${ }^{(32)}$.

Nesse contexto, entende-se que a avaliação constitui um recurso fundamental e potencial para compreender a qualidade do processo de ensinoaprendizagem, além da repercussão futura no cotidiano desses profissionais, ao influenciar beneficamente na disposição/disponibilidade na relação com usuários e na busca pela qualificação na área de drogas psicoativas, condizente aos diagnósticos identificados. Sobretudo, avaliar a satisfação dos participantes de cursos de formação tem sido considerado uma estratégia construtiva para o aprendizado do corpo docente e 
instituições, e para fundamentar novos planejamentos e currículos pretendidos em relação aos participantes do curso, e sua pertinência sociocultural(43).

A avaliação final ou mesmo o feedback que pode existir, durante toda a trajetória do participante/aluno, tende a indicar problemas e também a resolução, ajustando as dinâmicas de ensino e didáticas utilizadas( ${ }^{(17)}$. Mas, para que isso ocorra, é imprescindível a imparcialidade nos questionamentos/fichas, nos ambientes e oportunidades dessas avaliações não cerceando a participação de nenhum aluno e potencializando maior apontamentos sobre o desenvolvimento do curso(44).

Nesse sentido, a avaliação dos participantes sobre a proposta pedagógica de um curso não deve também possuir somente caráter de deliberação interna institucional, deve constar nos relatórios finais do curso e não se restringir à descrição sumarizada de dados, para que sirvam de embasamento para novas proposituras formativas ${ }^{(45-46)}$.

Como limitação do estudo, destaca-se a atualização do modelo de fichas durante as edições do curso, o que dificultou o levantamento dos dados e o agrupamento das informações. Ainda assim, o estudo revelou achados importantes, que irão auxiliar outras iniciativas semelhantes de formação de recursos humanos para atuação na área de drogas.

\section{Considerações finais}

O estudo apontou que, entre os participantes do curso, predominou a presença de pesquisadores do sexo feminino, provenientes do Brasil e do México, com profissões na área das ciências da saúde, principalmente enfermagem, atuando como docentes universitários. A maioria dos participantes produziu e publicou seus estudos científicos, gerados a partir do curso, dentro e fora do contexto brasileiro. Ao término das atividades da especialização, mostraram-se satisfeitos com o curso realizado em todos os aspectos.

O conhecimento dessas particularidades revelou algumas características dos profissionais da área e lacunas na sua formação. Igualmente, trouxe apontamentos para personalizar os processos formativos que vão desde o planejamento ao desenvolvimento e direcionamento das atividades de ensino-aprendizagem, em busca de propostas mais fidedignas às realidades desses profissionais. Esses subsídios poderão melhorar o planejamento dessa modalidade e desenho de curso, em edições futuras, ainda mais exitosas, seja em âmbito nacional como internacional, ao proporcionar aos participantes momentos de ampliação do conhecimento sobre o contexto das drogas, requisito necessário para a formação de pesquisadores nesta área.

\section{Referências}

1. United Nations Office on Drugs and Crime (UNODC). Drug Use and Health Consequences. N. 2: World Drug Report. [Internet]. Viena: UNODC; 2020 [cited 2020 Nov 20]. Available from: https://wdr.unodc.org/wdr2020/field/ WDR20_Booklet_2.pdf

2. Duarte PCAV, Formigoni MLOS. Efeitos de substâncias psicoativas: módulo 2. 7. ed. Brasília: Secretaria Nacional de Políticas sobre Drogas; 2014. 144 p.

3. Bokany V. Drogas no Brasil: entre a saúde e a justiça - Proximidades e opiniões. São Paulo: Editora Fundação Perseu Abramo; 2015.

4. Galvão AEO, Saavedra L, Cameira M. Economic and sociocultural poverty in drug abuse: from individual to sociopolitical responsibility. Saúde Soc. 2018;27(3). doi: http://doi.org/10.1590/S0104-12902018170970

5. Tatmatsu DIB, Siqueira CE, Prette ZAPD. Public policies for drug abuse prevention in Brazil and the United States. Cad Saúde Pública. 2020;36(1):e00040218. doi: https:// doi.org/10.1590/0102-311x00040218

6. Guo JL, Lee TC, Liao JY, Huang CM. Prevention of illicit drug use through a school-based program: results of a longitudinal, cluster-randomized controlled trial. J Adolesc Health. 2015;56(3):314-22. doi: https://doi. org/10.1016/j.jadohealth.2014.12.003

7. Nascimento MO, Micheli D. Avaliação de diferentes modalidades de ações preventivas na redução do consumo de substâncias psicotrópicas em estudantes no ambiente escolar: um estudo randomizado. Ciênc Saúde Coletiva. 2015;20(8). doi: https://doi. org/10.1590/1413-81232015208.15152014

8. Peres GM, Grigolo TM, Schneider DR. Desafios da Intersetorialidade na Implementação de Programa de Prevenção ao Uso Abusivo de Drogas. Psicol Cienc Prof. [Internet]. 2017 [Acesso 20 nov 2020];37(4):869-82. Disponível em: https://www.scielo.br/scielo.php?pid=S 141498932017000400869\&script=sci_arttext\&tlng=pt 9. Brasil. Lei no 11.343, de 23 de agosto de 2006 . Institui o Sistema Nacional de Políticas Públicas sobre Drogas - SISNAD; prescreve medidas para prevenção do uso indevido, atenção e reinserção social de usuários e dependentes de drogas; estabelece normas para repressão à produção não autorizada e ao tráfico ilícito de drogas; define crimes e dá outras providências. Brasília: Diário Oficial da União, 24 ago 2006 [Acesso 20 nov 2020]. Disponível em: http://www.planalto.gov.br/ccivil_03/_ ato2004-2006/2006/lei/l11343.htm

10. Coelho FJF, Monteiro ST. Desafios e Potencialidades de um Curso on-line sobre Drogas. EaD Foco. [Internet]. 2019 [Acesso 20 nov 2020];9(1):e742. Disponível em: https://eademfoco.cecierj.edu.br/index.php/Revista/ article/view/742/355

11. Matta ANB, Locatelli D, Silva EA, Contreras MV, Bittencourt BA, Noto AR. Processos formativos em álcool 
e outras drogas para trabalhadores da rede pública do município de São Paulo: a experiência do CRR-DIMESADUNIFESP. Pesqui Prát Psicossoc. [Internet]. 2016 [Acesso 5 set 2020];11(3):637-49. [Acesso 20 nov 2020]. Disponível em: http://www.seer.ufsj.edu.br/index.php/ revista_ppp/article/view/1962

12. Souza LF, Souza AC, Souza EO, Abrahão AL, Fernandes FL. A universidade como parceira na formação diferenciada para o cuidado aos usuários de drogas. Pesqui Prát Psicossoc. [Internet]. 2016 [Acesso 20 nov 2020];11(3):570-82. Disponível em: http://www.seer. ufsj.edu.br/index.php/revista_ppp/article/view/1949

13. Vecchia MD. Notas sobre a conjuntura da política de drogas no Brasil. J Health NPEPS. [Internet]. 2018 [Acesso 20 nov 2020];3(2):298-303. Disponível em: https://periodicos.unemat.br/index.php/jhnpeps/article/ view/3209

14. Coelho FJF, Monteiro S. Educação sobre drogas: possibilidades da EaD na formação continuada de professores. EaD Foco. [Internet]. 2017 [Acesso 20 nov 2020];7(2):2017. Disponível em: https://eademfoco. cecierj.edu.br/index.php/Revista/article/view/577/258 15. Maftum MA, Pagliace AGS, Borba LO, Brusamarello $T$, Czarnobay J. Changes in professional practice in the mental health area against Brazilian psychiatric reform in the vision of the nursing team. Rev Pesqui Cuidado Fundam Online. 2017;9(2):309-14. doi: http://dx.doi. org/10.9789/2175-5361.2017.v9i2.309-314

16. Sturmer G, Pinto MEB, Oliveira MMC, Dahmer A, Stein AT, Plentz RDM. Perfil dos profissionais da atenção primária à saúde, vinculados ao curso de especialização em saúde da família una-sus no Rio Grande do Sul. Rev Conhecimento Online. 2020;12(1):4-26. doi: https://doi. org/10.25112/rco.v1i0.1639

17. Viniegra RFS, Silva LGP, Aguiar AC, Souza L. Egressos de um Mestrado Profissional em Saúde da Família: Expectativas, Motivações e Contribuições. Rev Bras Educ Med. 2019;43(4):5-14. doi: https://doi. org/10.1590/1981-52712015v43n4rb20190078

18. Ribeiro IKS, Silveira RCP, Teixeira GS. Estilo de Vida y bienestar de Estudiantes Del área de la salud. Enferm Actual Costa Rica. 2017;(34):67-81. doi: http://dx.doi. org/10.15517/revenf.v0i34.30387

19. Ferreira FMPB, Mota LAN, Brito IS, Santos MR. Perfil de saúde dos estudantes de enfermagem: diagnóstico epidemiológico a partir do modelo PRECEDE-PROCEED. Rev Enferm Ref. 2017;4(15):91-100. doi: http://dx.doi. org/10.12707/RIV17047

20. Tuchman E, Hanley K, Naegle M, More F, Bereket S, Gourevitch MN. Integration and Evaluation of Substance Abuse Research Education Training (SARET) into a Master of Social Work program. Subst Abus. 2017;38(2):150-6. doi: http://doi.org/10.1080/08897077.2017.1291465
21. Conceição JM, Teixeira MRF. A produção científica sobre as mulheres na ciência brasileira. Rev Contexto Educ. 2020;35(112):280-99. doi: https://doi. org/10.21527/2179-1309.2020.112.280-299

22. Souza LM, Ávila MA. Mulheres na ciência e tecnologia: revisão de literatura. Rev Cient Tecnol Gest Hum. [Internet]. 2020 [Acesso 20 nov 2020];10(1). Disponível em: http://www.fatecguaratingueta.edu.br/revista/index. php/RCO-TGH/article/view/294n

23. Savage CL, Daniels J, Johnson JA, Kesten K, Finnell DS, Seale JP. The inclusion of substance use-related content in advanced practice registered nurse curricula. J Prof Nurs. 2017;34(3):217-20. doi: https://doi.org/10.1016/j. profnurs.2017.08.006

24. Branco FMFC, Farias FLS, Dutok CMS, Castelo Branco T Neto, Vargas D, Barroso TMMDA. Padrão do consumo de álcool entre trabalhadores de uma universidade pública brasileira. Rev Enferm Ref. [Internet]. 2019 [Acesso 20 nov 2020];4(22):85-96. Disponível em: http:// www.scielo.mec.pt/scielo.php?script=sci_arttext\&pi $\mathrm{d}=$ S0874-02832019000300009

25. Villegas-Pantoja MA, Méndez-Ruiz MD, Valle-Alonso MJ, García-Perales LA. Nursing Course on Drug Addiction and its Effects on Beliefs and Intentions: A Quasiexperimental Study. Aquichan. 2020;20(2):e2027. doi: https://doi. org/10.5294/aqui.2020.20.2.7

26. Nash AJ, Marcus MT, Cron S, Scamp N, Truitt M, McKenna Z. Preparing Nursing Students to Work With Patients With Alcohol or Drug-Related Problems. J Addict Nurs. 2017;28(3):124-30. doi: https://doi.org/10.1097/ JAN. 0000000000000175

27. Finnell DS, Savage $C L$, Hansen $B R$, Sanchez $M$, White $\mathrm{KM}$, Johnson JA, et al. Integrating substance use content in an "overcrowded" nursing curriculum. Nurse Educ. 2018;43(3):128-31. doi: https://doi.org/10.1097/ NNE. 0000000000000438

28. Batista CB, Vasconcelos MPN, Vecchia MD, Queiroz IS. A educação permanente em redução de danos: experiência do Curso de Atenção Psicossocial em Álcool e outras Drogas. Interface Comun Saúde Educ. 2019;23:e180071. doi: https://doi.org/10.1590/Interface.180071

29. Bauer TX, Volpato RJ, Nascimento VF, Rocha EM, Oliveira PR, Almeida MASO, et al. Drogas psicoativas no contexto de trabalho do professor de escola pública. J Health NPEPS. 2018;3(1):166-84. doi: http://dx.doi. org/10.30681/252610102922

30. Silva PMC, Galon T, Moura AAM, Volpato RJ, Zerbetto SR, Gonçalves AMS. Capacitação multiprofissional sobre drogas no contexto escolar: formação, saúde e educação. J Health NPEPS. 2019;4(1):182-99. doi: http://dx.doi. org/10.30681/252610103362

31. Silva RMDO, Luz MDAD, Fernandes JD, Silva LSD, Cordeiro ALAO, Mota LDSRD. Tornar-se especialista: expectativas dos enfermeiros portugueses após a 
realização do curso de especialização. Rev Enferm Ref. [Internet]. 2018 [Acesso 20 nov 2020];(16):147-54. Disponível em: http://www.scielo.mec.pt/scielo.php?pid= S087402832018000100015\&script=sci_arttext\&tlng=ess 32. Costa PHA, Mota DCB, Cruvinel E, Paiva FS, Gomide HP, Souza ICW, et al. Capacitação em álcool e outras drogas para profissionais da saúde e assistência social: relato de experiência. Interface. (Botucatu). 2014;19(53):395404. doi: https://doi.org/10.1590/1807-57622014.06077 33. Silva MAB, Abrahão AL. Política de Atenção Integral aos usuários de álcool e outras drogas: uma análise guiada por narrativas. Interface. (Botucatu). 2019;24. doi: https://doi.org/10.1590/interface.190080

34. Montagnero AV, Bassan G, Veloso L. Drogas: uma análise semântica dos estudos brasileiros. SMAD, Rev Eletrônica Saúde Mental Álcool Drog. [Internet]. 2019 [Acesso 20 nov 2020];15(4). Disponível em: http://www. periodicos.usp.br/smad/article/view/164012/157445

35. Lemes AG, Nascimento VF, Rocha EM, Almeida MASO, Volpato RJ, Luis MAV. Terapia Comunitária como cuidado complementar a usuários de drogas e suas contribuições sobre a ansiedade e a depressão. Esc Anna Nery. 2020;24(3):e20190321. doi: http://dx.doi. org/10.1590/2177-9465-EAN-2019-0321

36. Lemes AG, Rocha EM, Nascimento VF, Volpato RJ, Almeida MA, Franco SE, et al. Benefícios da terapia comunitária integrativa revelados por usuários de substâncias psicoativas. Acta Paul Enferm. 2020;33:eAPE20190122. doi: http://dx.doi.org/10.37689/ acta-ape/2020A001222

37. Melnyk BM, Gallagher-Ford L, Zellefrow C, Tucker $S$, Thomas B, Sinnott LT, et al. The First U.S. Study on Nurses' Evidence-Based Practice Competencies Indicates Major Deficits That Threaten Healthcare Quality, Safety, and Patient Outcomes. Worldviews Evid Based Nurs. 2017;15(1):16-25. doi: https://doi.org/10.1111/ wvn.12269

38. Cunha SM, Araujo RB, Bizarro L. Profile and pattern of crack consumption among inpatients in a brasilian psychiatric hospital. Trends Psychiatry Psychother. [Internet]. 2015 [cited Nov 20, 2020];37(3):12632. Available from: https://www.scielo.br/pdf/trends/ v37n3/2237-6089-trends-37-03-00126.pdf

39. Silva DL, Torrezan MB, Costa JV, Garcia APRF, Toledo VP. Perfil sociodemográfico e epidemiológico dos usuários de um centro de atenção psicossocial álcool e drogas. Rev Enferm Atenção Saúde. [Internet]. 2017 [Acesso 20 nov 2020];6(1). Disponível em: http://seer.uftm.edu.br/ revistaeletronica/index.php/enfer/article/view/1659/pdf 40. Quintana PB, Rosales C, Vivas Francesconi G, Novaes CC, Rocha VPS, Rocha TAH. Análisis de la producción de conocimiento de los egresados de la especialización en salud de la familia del Programa Mais Médicos. Rev Panam Salud Publica. [Internet]. 2020 [Acceso
20 nov 2020];44. Disponible en: https://iris.paho. org/bitstream/handle/10665.2/52152/v44e372020. pdf? sequence $=1$ \&isAllowed $=y$

41. Bernardo JF, Pinheiro BO, Mena-Chalco JP, Micheli D. Mapeamento de pesquisadores precursores da área de prevenção de drogas em contextos educacionais no Brasil. RECIIS. [Internet]. 2018 [Acesso 20 nov 2020];12(2). Disponível em: https://www.reciis.icict.fiocruz.br/index. php/reciis/article/view/1515

42. Monteiro ÉP, Gomide HP, Silveira OS, Ronzani TM. Curso de prevenção ao uso de drogas: Descrição e avaliação de satisfação. Estud Psicol (Natal). [Internet]. 2016 [Acesso 20 nov 2020];21(3):328-36. Disponível em: http://www.scielo.br/scielo.php?script=sci_ arttext\&pid=S1413-294X2016000300328\&Ing=en\&nr $\mathrm{m}=$ iso

43. Fonseca M, Fonseca DM. A gestão acadêmica da pósgraduação lato sensu: o papel do coordenador para a qualidade dos cursos. Educ Pesqui. 2016;42(1):151-64. doi: https://doi.org/10.1590/S1517-97022016031362633 44. Hypolito VAHA, Rosa SS, Luccas S. Avaliação pelos pares com o uso de tecnologias digitais no ensino superior. Rev Meta Aval. [Internet]. 2020 [Acesso 20 nov 2020];12(35):281-307. Disponível em: https:// revistas.cesgranrio.org.br/index.php/metaavaliacao/ article/view/2461/pdf

45. Freire JR Filho, Magnago C, Costa MV, Forster AC. Cursos de especialização ofertados no âmbito do Mais Médicos: análise documental na perspectiva da Educação Interprofissional. Interface. (Botucatu). [Internet]. 2018 [Acesso 20 nov 2020];22(suppl.2):1613-24. Disponível em: http://www.scielo.br/scielo.php?script=sci_ arttext\&pid $=$ S1414-32832018000601613\&Ing=en\&nr $\mathrm{m}=$ iso

46. Paulino VCP, Silva LA, Prado MA, Barbosa MA, Porto CC. Formação e saberes para a docência nos cursos de graduação em enfermagem. J Health NPEPS. [Internet]. 2017 [Acesso 20 nov 2020];2(1):272-84. Disponível em: https://periodicos.unemat.br/index.php/jhnpeps/article/ view/1822

\section{Contribuição dos autores}

Concepção e desenho da pesquisa: Margarita Antonia Villar Luis. Obtenção de dados: Margarita Antonia Villar Luis, Alisséia Guimarães Lemes. Análise e interpretação dos dados: Margarita Antonia Villar Luis, Alisséia Guimarães Lemes, Deivson Wendell Da Costa Lima, Liliane Santos Da Silva, Vagner Ferreira do Nascimento. Análise estatística: Alisséia Guimarães Lemes, Margarita Antonia Villar Luis. Redação do manuscrito: Margarita Antonia Villar Luis, Alisséia Guimarães Lemes, Deivson Wendell Da Costa Lima, Liliane Santos Da Silva, Vagner Ferreira do Nascimento. Revisão crítica do manuscrito quanto ao conteúdo intelectual importante: Margarita 
Antonia Villar Luis, Alisséia Guimarães Lemes, Deivson Wendell Da Costa Lima, Liliane Santos Da Silva, Vagner Ferreira do Nascimento.

Todos os autores aprovaram a versão final do texto. Conflito de interesse: os autores declararam que não há conflito de interesse. 